\title{
SINGLE TRANSISTOR OSCILLATOR BASED ON PIEZOELECTRIC RESONANT NANOCHANNEL FOR OPERATION IN LIQUIDS \\ Chiara Zuniga $^{l}$, Matteo Rinaldi ${ }^{2}$, Augusto Tazzoli ${ }^{3}$ and Gianluca Piazza ${ }^{3}$ \\ ${ }^{1}$ University of Pennsylvania, Philadelphia, Pennsylvania, USA \\ ${ }^{2}$ Northeastern University, Boston, Massachusetts, USA \\ ${ }^{3}$ Carnegie Mellon University, Pittsburgh, Pennsylvania, USA
}

\begin{abstract}
A single transistor oscillator based on an $18 \mathrm{MHz}$ piezoelectric resonant nanochannel is presented as the first demonstration of MEMS acoustic resonators with integrated nanochannels connected to a compact and portable oscillator readout enabling the sensor operation in liquids. This result represents a breakthrough for MEMS resonators operated in liquids as these devices are the first of their kind to be directly connected to a compact electronic oscillator.
\end{abstract}

\section{INTRODUCTION}

In recent years, the demand of miniaturized sensors able to operate in fluids has been steadily growing for a variety of different applications ranging from medical diagnostic to automotive and environmental monitoring.

The medical field, in primis, represents one of the most appealing and fast growing market for such sensors as the need to analyze human fluids recurs in almost every medical assessments. Particularly, the need of personalized and wireless medicine is becoming increasingly urgent.

Recently, miniaturized devices based on micro and nanoelectromechanical systems (M/NEMS) technology have emerged as a viable response to the need for compact, inexpensive and integrable resonant sensors. Their reduced mass allows them to achieve unprecedented levels of resolution [1] and makes them suitable for detecting minuscule concentrations of target analytes in bio-samples. However, the use of these devices for life science applications has been limited because of the challenge of operating them in liquid environments. Viscous damping, efficient transduction and effective fluid-device interaction represent the main issues that acoustic resonators need to overcome in order to be fully exploited for sensing applications in liquids.

Viscous damping, in particular, affects the performance of the NEMS devices depending on their mode of vibration and becomes increasingly detrimental as the size of the resonator is decreased.

Resonant devices vibrating according to flexural or out-ofplane bulk modes have shown greatly damped responses in fluids, with values of $Q$ in the order of 10 s - 100s [2-3]. In fact, in a fluid, these modes of vibration cause a pressure force on the displacing surfaces of the resonant device and creates additional damping with respect to shear-like vibrations. As a matter of fact, the latter are preferable for operation in liquids.

Thin Film Bulk Acoustic Resonators (FBARs) [4] have demonstrated similar performance in terms of quality factors but at much higher frequencies of operation, recording $\mathrm{Q}$ as high as 130 at $1.5 \mathrm{GHz}$. Therefore, they can provide values of mass sensitivity much higher than QCMs thanks to both their reduced volume and higher frequency of operation ( $\mathrm{GHz}$ range). Nevertheless, for FBAR, as for QCM, the operating frequency is set by the thickness of the piezoelectric layer. This precludes the capability of these devices to provide for multiple frequencies of operation (hence mass sensitivities) on the same substrate and exclude their employment for the fabrication of multi frequency single chip sensor arrays.

The use of laterally vibrating devices $[5,6]$ instead of devices vibrating in a flexural or out of plane bulk modes has shown to partially overcome some of these issues. They can provide for multiple frequencies on the same chip, and their interaction with the fluid is equivalent to shear vibrations, which are less prone to viscous damping as shear waves rapidly decay in fluid media.

The integration of a microfluidic channel inside the body of a resonant cantilever beam sensor $[1,6]$ has been demonstrated as a very effective way to simultaneously address the issue of viscous damping and realizing a compact delivery system that reduces sample volume. Although highly effective in maintaining unaltered mechanical performances, these hollow cantilever beams required a high DC voltage (as high as 120V [1]) for their electrostatic actuation and a cumbersome optical readout. In addition, they exhibited a frequency of operation of few 100s of $\mathrm{kHz}$, which limited the device sensitivity for certain applications.

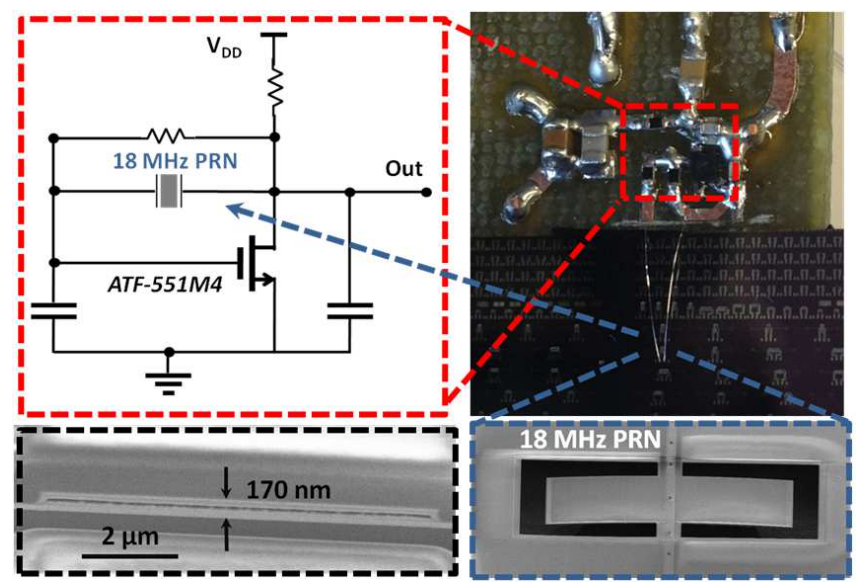

Figure 1: SEM of the $18 M \mathrm{MH}$ PRN and its cross-section and a picture of the device wire-bonded to a Pierce oscillator implemented with an ATF-551M4 E-pHEMT GaAs transistor.

This paper presents a new design and the experimental verification of Piezoelectric Resonant Nanochannels (PRNs). The reduction of viscous damping has been attained through an innovative design solution, in which the fluid is enclosed in the body of the resonant element. In this perspective, the hollow design of a resonant nanochannel implements a high surface to volume sensor that maximizes the sample-device interaction, simultaneously solves the issues related to the transduction efficiency of nanoscale structures in liquids, and addresses the reduction of viscous damping.

PRNs with frequencies of operation ranging from $12 \mathrm{MHz}$ to $1 \mathrm{GHz}$ were designed, fabricated on the same die and tested achieving quality factors as high as 1700 when operated in fluids at $13 \mathrm{MHz}$. Several designs and channels widths have been designed and experimentally tested in order to find the best trade-off between sensitivity and quality factors.

Finally, the AIN PRNs can be efficiently actuated and sensed piezoelectrically on-chip (values of electromechanical coupling, $k_{t}^{2}$, as high as $1 \%$ are achieved despite the operation in fluid 
environment) solving the fundamental transduction issues associated with M/NEMS sensors in fluids and enabling the use of compact and low power CMOS circuits for electronic readout. The capability to connect an $18 \mathrm{MHz}$ PRN to self-sustained, lowpower, single transistor oscillator circuits for direct frequency readout is experimentally demonstrated. The fabricated oscillators show excellent noise performance (phase noise values as low as $-110 \mathrm{dBc} / \mathrm{Hz}$ at $1 \mathrm{kHz}$ offset and phase noise floor values as low as $-154 \mathrm{dBc} / \mathrm{Hz}$ are achieved with a carrier frequency of $\sim 18 \mathrm{MHz}$ ) thanks to the outstanding and unique performance of the nanoscaled resonant elements in terms of figure of merit $(F o M)$, corresponding to a $k_{t}^{2} \cdot Q$ product as high as 7 despite operation in fluids. Because of the unique capability of arraying large number of PRNs with different frequencies of operation in a small footprint, this novel technology lays the foundation for the demonstration of large arrays of Piezoelectric Resonant Nanochannels for highly multiplexed bio-sensing applications that require high throughput and reduced sample volume.

\section{DESIGN}

The device that constitutes the basis of this work consists of a laterally vibrating nanochannel [7, 8]. As explained elsewhere [8], the Piezoelectric Resonant Nanochannel is composed of two layers of AlN enclosing a nano-scaled airgap in between them. Such airgap serves as a nanofluidic channel whose function is to confine a liquid sample within the body of the device instead of having the resonant sensors submersed in it.

A PRN fully displaces into contour extensional vibrations when an electric field is applied across the thickness of the first AlN film as displayed in Figure 2 in the same way as a conventional CMR [9].

The improved design solution presented in this paper delivers the fluid solely on a narrow area of the resonator, a fraction of the total surface of the device in the range from $3.3 \%$ to $30 \%$. Thus, this minimizes the energy radiation and translates to an improved $Q$. The devices are also properly designed to span an extended frequency range from $12 \mathrm{MHz}$ to $1 \mathrm{GHz}$ thus benefiting from the theoretical $Q$ enhancement at both ends of the range. Furthermore, because of the additional confinement of the fluidic sample into a narrower area on the $\mathrm{PRN}$, the expression for the $Q$ in fluids previously computed in [5] needs to take into account that the area in contact with the liquid, where the energy radiation occurs because of the viscous drag, is now much smaller than the PRN top surface as shown in Figure 2.
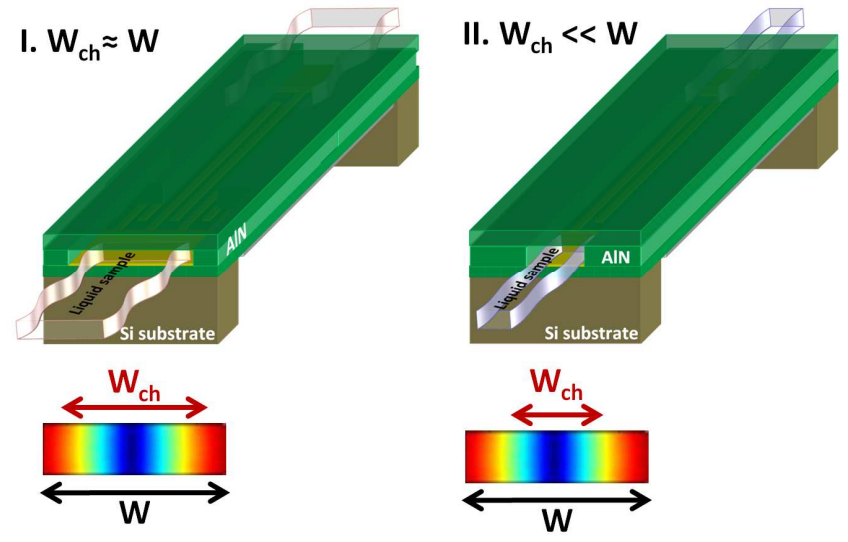

Figure 2: Schematic $c$ of the new comparison design solution of the PRN (II.) that confines the fluid to a narrower area on the device's body
The expression of the $\mathrm{Q}$ in fluids therefore becomes

$$
Q_{f}=\omega_{s} \cdot \frac{\rho_{e q} \cdot T_{e q} \cdot u_{0}}{2 \operatorname{Re}\left[\sigma_{x y}\right]} \frac{S}{S_{\text {channel }}}
$$

where $S_{\text {channel }}$ is the internal area of the nanochannel and $S$ the surface of the PRN, while all the remaining variables $T_{e q}, \rho_{e q}, \sigma_{x y}$ are respectively the equivalent thickness and the equivalent density of the material stack forming the device, and the frictional force acting on the device surface.

Intuitively, it is straightforward to realize that by minimizing the area where the energy dissipation occurs represents an alternative way to enhance the quality factor without further scaling the nanochannel depth to extremely reduced thicknesses. Additional scaling would, in fact, add significant complexity to the fabrication process and would result in nanochannels with values of flow resistance so high to render the connection to a complete flow system almost unfeasible.

Figure 3 is a plot of Eq. 1 and clearly predicts that there is a significant advantage in designing narrow nanochannels operating over the whole frequency range.

The location of the nanochannel within the body of the PRN is an additional design parameter and has a significant weight on the performance of the mechanical resonator. In fact, the induced displacement of the resonator is not uniform over its entire width, since the mode-shape of vibration obeys to a harmonic motion, $u(x)$ [9]. Therefore, designing the nanochannel in the locations of maximum displacement of the PRN translates to higher mass sensitivity, but at the same time higher viscous damping and hence, lower quality factor. Conversely, placing the nanochannel in a nodal point, i.e. where the surface velocity is zero, would noticeably increase the quality factor when the nanochannel is filled, but it would also dramatically decrease the mass sensitivity of the device.

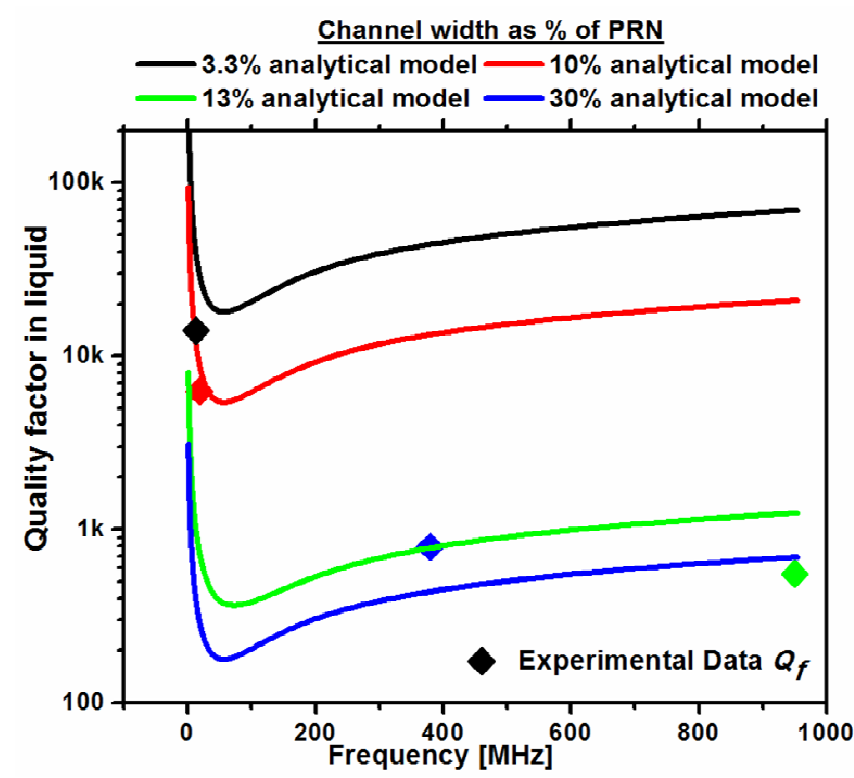

Figure 3: The graph shows the overlap between the experimental Qs in DI-water extracted from the fitted admittance curves and the predicted behavior according to the theory of a laterally oscillating plate in viscous fluids where $1 / Q_{\text {tot }}=1 / Q_{\text {fluid }}+1 / Q_{\text {air }}$ with $Q_{\text {fluid }}$ being the fluidic quality factor and $Q_{\text {air }}$ the quality factor of the PRN in air. The data show good agreement with the theory especially for the lower frequency devices (single finger devices) 
where the hypothesis of uniform velocity of the plate surface represents a good approximation

Based on these considerations, Eq. 1 of the quality factor in fluids should be modified to take into account the location of the channel. As a matter of fact, the lower frequency devices consist of a single finger (for which the width, $W$, is between 200 and 300 $\mu \mathrm{m})$ vibrating in the fundamental extensional mode and the nanochannel runs solely on top of the center of the structure. This channelhas a width, $W_{c h}$, considerably smaller than the one of the device ( $W_{c h}$ is $\left.10-20 \mu \mathrm{m}\right)$ (Fig. 2-II). For this reason, the velocity of the internal surface of the nanochannel cannot be considered to be maximum, but in light of the fact that $W_{c h} / W \rightarrow 0$, it can be approximated as $u\left(W_{c h}\right) \approx u_{0} \frac{\pi W_{c h}}{2 W}$. Hence, the expression for the quality factor is:

$$
Q_{f}=\omega_{s} \cdot \frac{\rho_{e q} \cdot T_{e q}}{\operatorname{Re}\left[\frac{\sigma_{x y}}{u_{0}}\right]} \frac{2 W^{3}}{\pi^{2} W_{c h}{ }^{3}}
$$

The effect of these design changes on sensitivity and limit of detection (LOD) should also be taken into consideration. In fact, the expression of the mass sensitivity for these PRNs vibrating at low frequency $(12-50 \mathrm{MHz})$ needs to take into account the reduction in both velocity and sensing area inside the nanochannel. Therefore, the traditional sensitivity equation [5] can be modified as follows

$$
S=\frac{f_{0}}{2 \rho_{e q} T_{e q}} \frac{\pi^{2} W_{c h}{ }^{3}}{12 W^{3}}
$$

Finally, the ultimate limit of detection, $L O D$, of a PRN given its mass sensitivity, $S$, computed in Eq. (3) can be derived considering the resonator thermomechanical noise as the ultimate source of frequency fluctuations originated from thermally driven random motion of the mechanical device [11]. In this case, the LOD can be expressed as:

$$
L O D=\frac{\Delta f_{\min }}{S}=\sqrt{\frac{k_{B} T(1+F) B}{P_{c}}} \frac{\rho T}{Q} \frac{12 W^{3}}{\pi^{2} W_{c h}{ }^{3}}
$$

where for the higher frequency devices, for which $Q$ is effectively limited by $Q_{f}$ in liquids, it is possible to substitute Eq. 2 in the LOD equation and obtain an expression of the limit of detection that is a function of the dissipative part of the frictional force and no longer dependent on the nanochannel in plane dimensions. At lower frequency, for which the $\mathrm{Q}$ is likely limited by the intrinsic material damping (see Figure 3), there is a net advantage in miniaturizing the lateral dimensions of the channel.

\section{EXPERIMENTAL RESULTS}

The new design has demonstrated performance far superior than the first generation of PRNs effectively proving the enhancement in both the design and fabrication process $[7,8]$. The confinement of the nanochannel over a fraction of the total surface of the device together with a nanochannel thickness comparable to the penetration depth in water $(\sim 160 \mathrm{~nm}$ at $13 \mathrm{MHz})$ allowed the PRNs with very narrow channels' widths $(3.3 \%$ to $10 \%$ of the device total width) to attain $Q s$ as high as 1710 at $13 \mathrm{MHz}$ and equal to 1567 at $18.6 \mathrm{MHz}$ when filled with DI-water. Despite the operation in liquid environment, a high value of the figure of merit, $k_{t}^{2} \cdot Q,(\sim 10$ and 9 at 13 and $18 \mathrm{MHz}$, respectively) have been attained translating to very low values of motional resistance.

Such high values of the device $k_{t}^{2} \cdot Q$ have enabled the direct connection of the fabricated $18 \mathrm{MHz}$ PRN to a compact and low power self-sustained oscillator circuit for direct frequency readout.

The response of an $18 \mathrm{MHz}$ PRN with $10 \%$ channel coverage has been characterized through a RF probe station recording Qs as high as 1600 and 1420, respectively in air and when filled with DIwater. Unchanged values of $k_{t}^{2}$ equal to $0.6 \%$ have been achieved.

The device was directly wire-bonded to a Pierce oscillator implemented with an ATF-551M4 E-pHEMT GaAs transistor (Figure 1). The oscillator was tested for different biases (i.e. different power levels) and its phase noise (PN) monitored with an Agilent E5052B Signal Source Analyzer.

The output of the oscillator in both operating conditions, air and DI-water, was also recorded with a Spectrum Analyzer. Selfsustained oscillations were observed for values of bias voltages, $\mathrm{V}_{\mathrm{DD}}$, in the range of $1.8 \mathrm{~V}$ and $3.4 \mathrm{~V}$.

The oscillator achieved values of $\mathrm{PN}$ as low as $-110 \mathrm{dBc} / \mathrm{Hz}$ at $1 \mathrm{kHz}$ and a noise floor of $-154 \mathrm{dBc} / \mathrm{Hz}$. After a short transient when water initially fills the channel (Figure 5) these values are nearly unchanged whether the nanochannel is filled with air or DIwater.

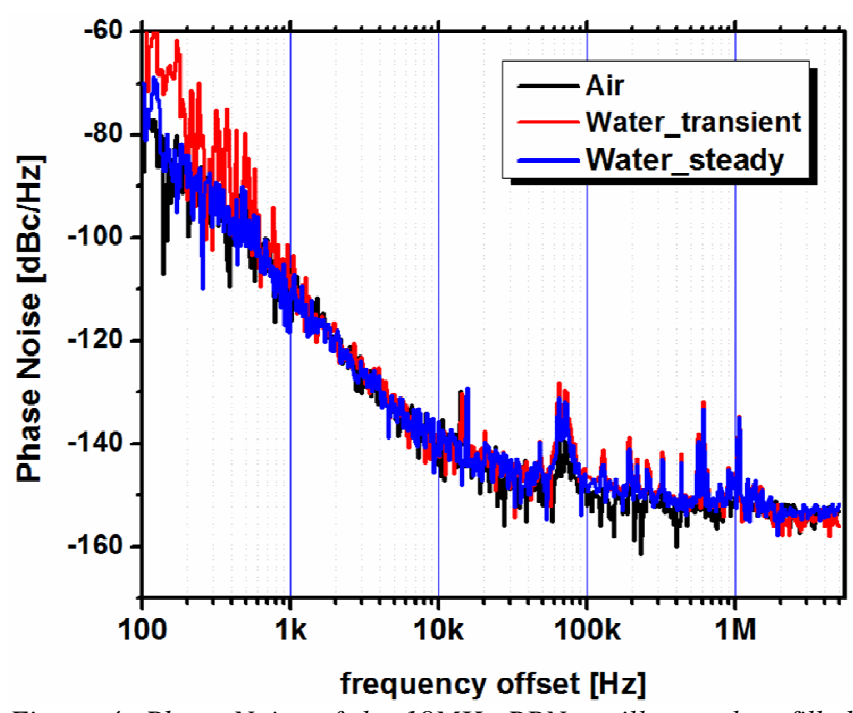

Figure 4: Phase Noise of the 18MHz PRN oscillator when filled with air and water. When water is first filling the channel, a transient response associated to higher close-in phase noise can be noticed. Unchanged performances are shown for both operating conditions when transient associated with water diffusion inside the channel is over.

Given the device phase noise, it is possible to compute through a simple mathematical integration the Allan variance, a statistical tool commonly used to measure the time domain frequency fluctuation of oscillators for a given integration time, $\tau$ (measurement time). Unlike the phase and frequency noise that are defined in the frequency domain, the Allan variance is defined in the time domain as the variance over time in the measured frequency of a source, each measurement averaged over a time interval, $\tau$, with ideally no dead time between measurement intervals.

The Allan variance is related to phase noise through the following relationship:

$$
\sigma_{Y}^{2}(\tau)=\frac{2}{\pi^{2} \tau^{2}} \int_{0}^{\infty} S_{\phi, o}(\omega) \sin ^{4}(\pi \omega \tau) d \omega
$$

where $S_{\phi, o}(\omega)$ is the oscillator phase noise and $\omega$ is the offset 
frequency from carrier. For the form of the close-in phase noise density, the Allan deviation is theoretically described by:

$$
\sigma_{Y}(\tau) \approx \sqrt{\frac{k_{B} T_{0}(1+F) \omega_{0}^{2}}{4 P_{c} Q^{2} \tau}}
$$

Therefore, by applying Eq. (5) to the phase noise experimentally recorded for the $18 \mathrm{MHz}$ PRN oscillator, it is possible to plot the Allan deviation of the oscillator and extract the experimental minimum detectable frequency shift in both operating conditions.

Figure 5 shows the Allan Deviation for the $18 \mathrm{MHz}$ PRN oscillator extracted from the phase noise measurements when the device is operated in air and during the transient response, when water is initially filling the nanochannel. The latter situation represents the worst case scenario, since at steady state the PRN filled with water presents the same noise performance as if operated in air as clearly shown in Figure 5.

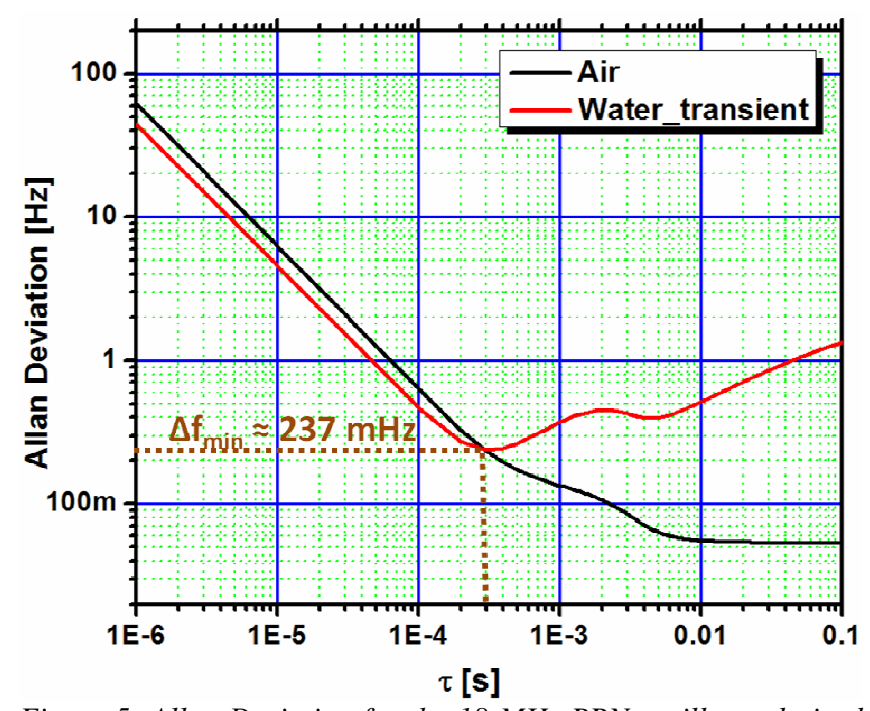

Figure 5: Allan Deviation for the $18 \mathrm{MHz}$ PRN oscillator derived from the phase noise in both operating conditions

A minimum detectable frequency shift equal to $237 \mathrm{mHz}$ has been extracted from the Allan deviation of the water transient curve for a measurement time of roughly $500 \mu \mathrm{s}$. Using Eq. (4) and the sensitivity computed for the $18 \mathrm{MHz}$ PRN, this can be translated to a value of limit of detection equal to $4 \mathrm{ag} / \mu \mathrm{m}^{2}$, which computing the internal surface of the nanochannel of the $18 \mathrm{MHz}$ $\mathrm{PRN}$, is equivalent to an effective absolute mass of $4.8 \mathrm{fg}$. Nevertheless, such experimental value is still slightly higher than the theoretical limit of detection (LOD) for a PRN limited by thermomechanical fluctuations as computed in Eq. 4. The experimentally recorded phase noise and derived Allan Variance are in fact not limited by the thermomechanical noise of the resonator, but the flicker noise of the oscillator. The theoretical thermomechanical limit is instead equal to $0.115 \mathrm{ag} /\left[\mu \mathrm{m}^{2} \cdot \sqrt{\mathrm{Hz}}\right]$, or to $1.6 \mathrm{ag} / \mu^{2}$ for a measurement time of $500 \mu \mathrm{s}$ and $3.0 \mathrm{~V}$ bias voltage $\left(V_{D D}\right)$. This clearly implies that if the read out circuit is properly improved, the LOD could be further lowered. Additionally, the power level $P_{C}$ used to drive the PRN at its center frequency could be also increased to achieve better signal to noise ratio and, hence, lower limit of detection.

\section{CONCLUSIONS}

In this paper, a new and improved design of Piezoelectric
Resonant Nanochannels was experimentally demonstrated. Qs as high as 1710 at $13 \mathrm{MHz}$ and equal to 1567 at $18.6 \mathrm{MHz}$ when filled with DI-water, were attained.

Thanks to such high values of the devices figure of merit, the capability to connect a $18 \mathrm{MHz}$ PRN to low-power single transistor oscillator circuit for direct frequency readout was experimentally demonstrated. The fabricated oscillator shows excellent noise performance: phase noise values as low as $-110 \mathrm{dBc} / \mathrm{Hz}$ at $1 \mathrm{kHz}$ offset and phase noise floor values as low as $-154 \mathrm{dBc} / \mathrm{Hz}$ were achieved for a carrier frequency of $\sim 18 \mathrm{MHz}$ ).

Additionally, a minimum detectable frequency shift equal to $237 \mathrm{mHz}$ has been extracted from the Allan deviation of the transient response of a device filled with DI water and a measurement time of roughly $500 \mu \mathrm{s}$. This can be translated to a value of limit of detection equal to $4 \mathrm{ag} / \mu \mathrm{m}^{2}$, which corresponds to a total mass of $4.8 \mathrm{fg}$.

This result represents a breakthrough for MEMS resonators operated in liquids as these devices are the first of their kind to be directly connected to a compact electronic oscillator.

\section{ACKNOWLEDGMENTS}

The authors wish to thank the staff of the Wolf Nanofabrication Facility (WNF) at the University of Pennsylvania. This work was supported by NTI.

\section{REFERENCES}

[1] J. Lee, W. Shen, K. Payer, T. P. Burg, and S. R. Manalis, "Toward Attogram Mass Measurements in Solution with Suspended Nanochannel Resonators" Nano Letters, 2010, 10, pp. 2537-2542.

[2] S. Choi, W. Xu, X. Zhang and J. Chae, " Characterization of a High-Q in-liquid Longitudinal-Mode Film Bulk Acoustic Resonator for Real-Time in-situ Monitoring of Competitive Protein Adsorption", Proceedings of IEEE MEMS 2010, pp. 739 742.

[3] M. Yue, H. Lin, D. E. Dedrick, S. Satyanarayana, A. Majumdar, A. S. Bedekar, J. W. Jenkins, and S. Sundaram, "A 2-D Microcantilever Array for Multiplexed Biomolecular Analysis", Journal of Microelectromechanical Systems, Vol. 13, No. 2, April 2004.

[4] M. Yue, J. C. Stachowiak, H. Lin, R. Datar, R. Cote, and A. Majumdar, "Label-Free Protein Recognition Two-Dimensional Array Using Nanomechanical Sensors", Nano Letters, 2008 Vol.8, No.2, pp. 520-524.

[5] C. Zuniga, M. Rinaldi and G. Piazza, "Quality Factor of MEMS and NEMS AlN Contour Mode Resonators in Liquid Media", IEEE International Ultrasonic Symposium Proceedings 2009, pp. 2568-2571.

[6] R. Cox, F. Josse1, S. Heinrich, I. Dufour and O. Brand, "Resonant Microcantilevers Vibrating Laterally in Viscous Liquid Media" IEEE International Frequency Control Symposium, 2010.

[7] C. Zuniga, M. Rinaldi and G. Piazza, "High Frequency Piezoelectric Resonant Nanochannel for Bio-Sensing Applications in Liquid Environment" Proceedings IEEE Sensors 2010, pp. 5255.

[8] C. Zuniga, M. Rinaldi and G. Piazza, "Reduced viscous damping in High Frequency Piezoelectric Resonant Nanochannels for Sensing in Fluids", Proceedings of IEEE MEMS 2011, pp. 960963.

[9] M. Rinaldi, C. Zuniga, N. Sinha, M. Taheri, S. M. Khamis, A. T. Johnson, and G. Piazza, "Gravimetric Chemical Sensor Based on the Direct Integration of SWNTs on AlN Contour-Mode MEMS Resonators", Proceedings of the IEEE International Frequency Control Symposium 2008, pp, 443-448. 